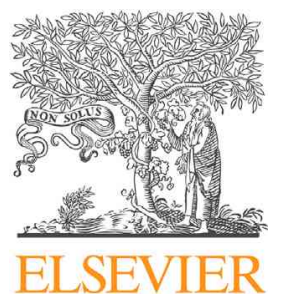

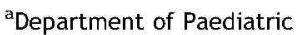
Urology, Royal Manchester Children's Hospital, UK

${ }^{\mathrm{b}}$ Institute for Surgical Research, University of Szeged, Hungary

'Department of Paediatric Surgery, Royal Manchester Children's Hospital, UK

Correspondence to: T. Cserni, Department of Paediatric Urology, Royal Manchester Children's Hospital, UK

tcserni@yahoo.com (T. Cserni)

\section{Keywords}

Alternative Mitrofanoff; Spiral intestinal lengthening (SILT); orthogonal polarising spectral imaging (OPS)

Received 19 August 2014 Accepted 26 January 2015 Available online 11 March 2015

\title{
New alternative Mitrofanoff channel based on spiral intestinal lengthening and tailoring
}

\author{
Raimondo M. Cervellione ${ }^{\mathrm{a}, \mathrm{b}}$, Daniel Hajnal ${ }^{\mathrm{b}}$, Gabriella Varga ${ }^{\mathrm{b}}$, \\ George Rakoczy ${ }^{c}$, Rainer Kubiak ${ }^{c}$, Jozsef Kaszaki ${ }^{b}$, Mihaly Boros ${ }^{b}$, \\ Rachel Harwood ${ }^{a}$, Alan P. Dickson ${ }^{a}$, Tamas Cserni ${ }^{\text {a,b }}$
}

Summary

\section{Introduction}

The occasional lack of appendix and the increasing use of the Malone anterograde continence enema (MACE) procedure have expanded the need for alternative Mitrofanoff channels. The Monti procedure does not always provide adequate length, the anastomosis of the double Monti, and the potential kink of the Casale channel is not ideal for smooth catheterisation. We tested the concept of spiral intestinal lengthening and tailoring (SILT), we developed originally for short bowel syndrome, to create a long and straight alternative Mitrofanoff channel (Figure).

\section{Material and methods}

After ethical approval five mini-pigs underwent spiral intestinal lengthening and tailoring (SILT) without any previous bowel dilatation procedure. (Mean bowel width was $20.5 \pm 0.57 \mathrm{~mm}$ ). The spiral line was marked on a $6-8-\mathrm{cm}$-long ileum approximately $15 \mathrm{~mm}$ apart with a $60^{\circ}$ angle to the longitudinal axis of the bowel. When the incision was completed, the mesentery was incised perpendicularly where the spiral incision line met the mesentery. The maximum length segment hanging on a single $1.5-\mathrm{cm}$-wide well-vascularised mesentery was detached. The capillary red blood cell velocity (RBCV) and perfusion rate (PR) was measured at the edges of the opened bowel strip by in vivo microscopy using orthogonal polarising spectral imaging
(Cytoscan A/R, Cytometrics, Philadelphia, PA, USA). The bowel strips have been reconstructed in spiral fashion over a $12 \mathrm{~F}$ catheter and were implanted into the bladder. Viability, patency, and microcirculation were assessed 4 weeks later. Conventional microscopy with HE staining was performed.

\section{Results}

The mean length of the spiral channel $(100 \pm 26.4 \mathrm{~mm})$ was longer than could have been achieved with the double Monti or Casale procedure (4 times the bowel width). A $17 \%$ and $8.3 \%$ reduction was measured in the median values of the RBCV and the PR at the edges of the bowel strip at the primary surgery. All implanted channels remained viable, straight, patent, and easily catheterisable after 4 weeks, with full recovery of the RBCV and PR. The histology showed no necrosis or fibrosis.

\section{Conclusion}

The SILT concept is suitable for creating a long and straight alternative Mitrofanoff channel.

\section{Discussion}

However, the SILT technique has been reported to be successful in the clinical practice to tailor and lengthen dilated short bowel; in this study we first applied this technique on normal calibre intestine to create long alternative Mitrofanoff channel. The use of an animal model and the relative short-term observation are the limitations of this study.

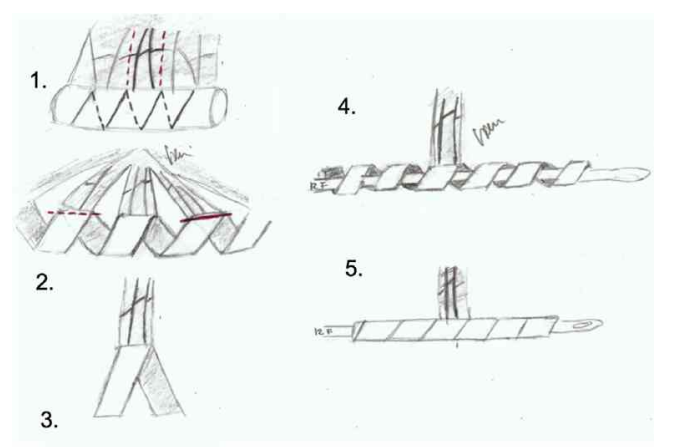

Figure Procedure steps. 1. The spiral incision line on the ileum and incision on mesentery. 2. A segment with the mesentery in the middle disconnected (incision at the dotted lines). 3 . The disconnected bowel strip hanging on the mesentery. 4. Creation of a Mitrofanoff channel in a spiral fashion above a catheter. 5 . The reconstructed channel. 


\section{Introduction}

The appendix has been used most commonly as a catheterisable stoma by paediatric urologists and reconstructive surgeons since Mitrofanoff described it in 1980 [1]. There are several benefits of using the appendix as a catheterisable stoma. The mesentery supplies the appendix in the middle portion and therefore the two ends are not bulky, thus making implantation into the bladder easy. It is long enough to be implanted into the bladder in an antireflux manner and to reach the skin level through the abdominal wall. It is very important that it is naturally straight and narrow to smoothly guide catheters into the bladder.

The occasional lack of a suitable appendix and the increasing use of the MACE procedure have expanded the need to find alternative tissues for the Mitrofanoff channel.

The Monti channel, transversally tubularised ileum, is a well-known alternative [2]. The length of the Monti channel is determined by the diameter of the ileum. This may be shorter than the appendix and does not always provide adequate channel length to implant it into the bladder and traverse the abdominal wall without tension.

The demand of longer channels resulted in alternative techniques such as double Monti and Casale tubes. The circular anastomosis in the double Monti and the significant angulation in the middle of the Casale channel, which has to be straightened surgically during its creation, may be associated with higher complication rates [3,4].

The authors propose the application of the concept of spiral intestinal lengthening and tailoring (SILT) developed by their team to create a long and straight viable alternative the Mitrofanoff channel [5-7].

\section{Material and methods}

The study was performed on anaesthetised female Vietnamese mini-pigs ( $n=5$, weight: $25-30 \mathrm{~kg}$ ). The animals were kept under conventional circumstances, in standard cages, and fed with commercially available mixed food; they were fasted for $36 \mathrm{~h}$ before surgery and always had free access to water. Anaesthesia was induced with an intramuscular injection of a mixture of ketamine $(20 \mathrm{mg}$ / $\mathrm{kg})$ and xylazine $(2 \mathrm{mg} / \mathrm{kg})$ and maintained with a continuous infusion of propofol ( $2 \% ; 50 \mu \mathrm{L} / \mathrm{kg} / \mathrm{min}$ iv). An endotracheal tube was inserted and the animals were ventilated mechanically with a volume-controlled ventilator. The tidal volume was set at $8-9 \mathrm{~mL} / \mathrm{kg}$, and the respiratory rate was adjusted to maintain the end tidal carbon dioxide pressure between 35 and $45 \mathrm{mmHg}$. Norocarp S (carprofen; $4 \mathrm{mg} / \mathrm{kg}$ ) and normal saline infusion were administered via a cannulated ear vein. Heart rate, $\mathrm{O}_{2}$ saturation, $\mathrm{PCO}_{2}$, and body temperature were continuously monitored by capnometry.

Median laparotomy was performed after skin preparation with povidone-iodine (Betadine, Egis, Budapest, Hungary) and sterile draping. Clam ileocystoplasty was performed. Another $6-8-\mathrm{cm}$-long piece of ileum adjacent to the ileal bladder flap was mobilised with intact mesenteric blood supply. The spiral line was marked on the bowel approximately $15 \mathrm{~mm}$ apart with a $60^{\circ}$ angle to the longitudinal axis of the bowel. When the incision was completed, the mesentery was incised perpendicularly where the spiral incision line met the mesentery. The maximum length segment hanging on a single $1.5-\mathrm{cm}$-wide well-vascularised mesentery was detached. The microcirculation was recorded in the middle and at the edges of the bowel strip.

The bowel strips were then retubularised in a spiral fashion with interrupted $5 / 0$ Vicryl sutures over a $12 \mathrm{~F}$ Malecoth catheter and were implanted in the bladder according to the Mitrofanoff principle. The distal end of the channels were brought out through the abdominal wall, sutured to the skin, and secured to the fascia and peritoneum. The catheters were secured to the skin with 5/ 0 vicryl and were cut at the level of the skin. Another $12 \mathrm{~F}$ Malecoth catheter was inserted into the urethra, cut at the level of the Rima pudendi, and secured into the bladder mucosa and labia minora with $5 / 0$ Vicryl stitches. The steps to create a catheterisable channel are demonstrated in Fig. 1.

Four weeks later the animals were anaesthetised again and the catheterisable channels were examined and catheterised. Laparotomy was performed, the channels incised, and mucosal microcirculation was recorded at the level of the bladder, adjacent to the abdominal wall and in the middle at the level of the mesentery. The channels were removed in one piece with the bladder for histological examination. Paraffin-embedded cross-sections of the channels at the level of the bladder, adjacent to the skin, in the middle, and at the level of the mesentery were stained with haematoxylin eosin for conventional histology.

\section{Microcirculation analysis}

The intravital orthogonal polarisation spectral (OPS) imaging technique (Cytoscan A/R, Cytometrics, Philadelphia, PA, USA) was used for visualisation of the microcirculation of the mucosa [8]. The microscopic images were recorded with an S-VHS video recorder (AG-TL 700, Panasonic, Kadoma, Japan). Microcirculatory evaluation was performed off-line by frame-to-frame analysis of the videotaped images. The capillary red blood cell velocity (RBCV, $\mu \mathrm{m} / \mathrm{s}$ ) changes in the post-capillary venules and the capillary perfusion rate (ratio of perfused capillaries and total capillaries) were determined in three separate fields by means of a computer-assisted image analysis system (IVM Pictron, Budapest, Hungary). All microcirculatory evaluations were performed by two independent investigators. Data analysis was performed with a statistical software package (SigmaStat for Windows, Jandel Scientific, Germany). Differences between groups were analysed with the Kruskal-Wallis one-way analysis of variance on ranks, followed by Dunn's method for pairwise multiple comparison. In Fig. 2, median values and 75th and 25th percentiles are given; $p<0.05$ was considered significant.

\section{Results}

The mean width of the ileum was $20.5 \pm 0.57 \mathrm{~mm}$. The authors were able to create straight spiral channels over a $12 F$ Foley catheter with a mean length of $100 \pm 26.4 \mathrm{~mm}$.

The RBCV dropped $17 \%$ at the edges of the bowel strip after the spiral incision to a median $397,171 \mu \mathrm{m} / \mathrm{s}(25 \%$ : 


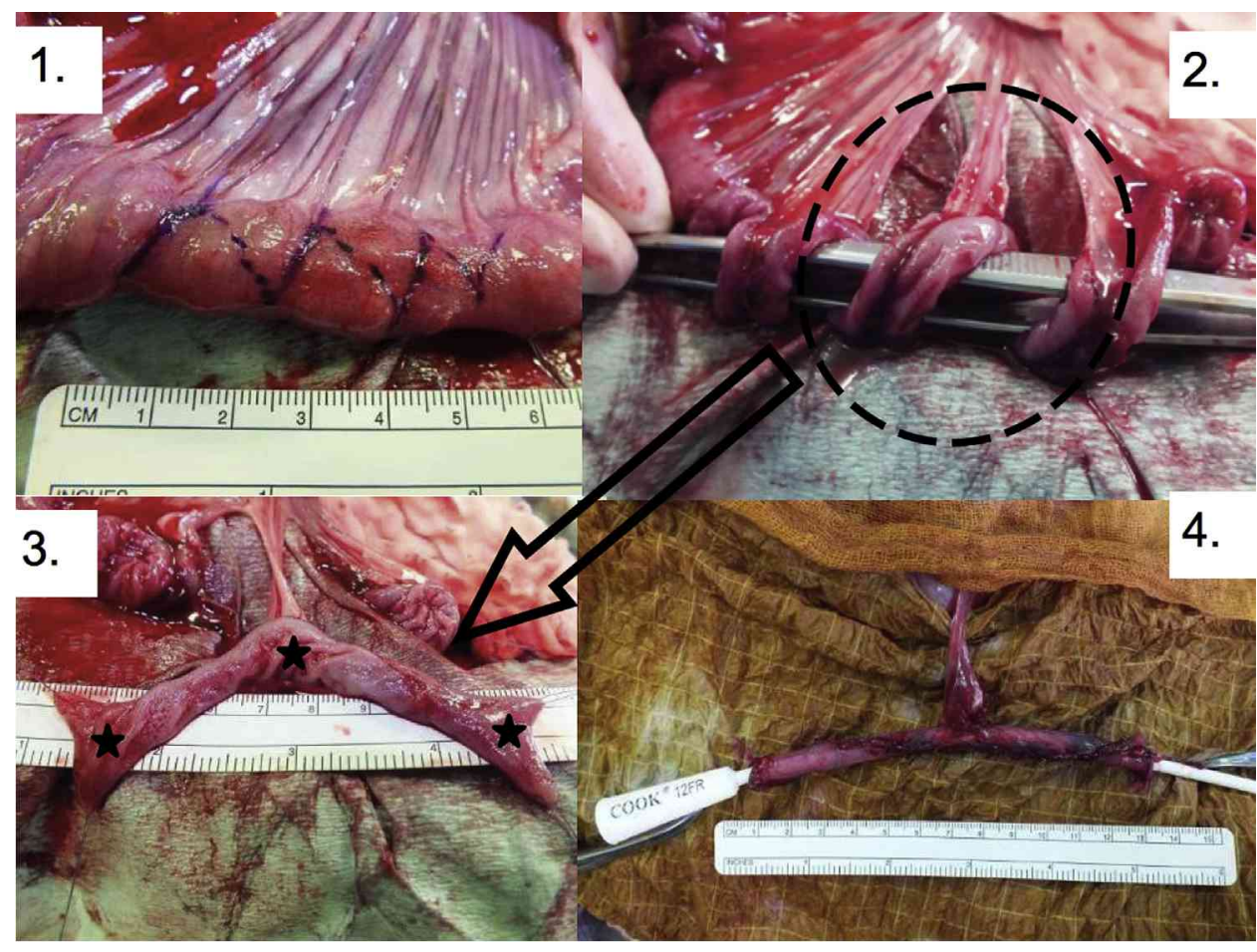

Figure 1 Procedure steps. 1. Spiral incision line on the ileum and incision on mesentery. 2. A segment with the mesentery in the middle disconnected. 3 . The disconnected bowel strip on the mesentery; the stars mark the points where microcirculation was measured. 4 . The reconstructed channel.

322,016, 75\%: 444,652). This difference was statistically significant compared with control values: median 478,846 $\mu \mathrm{m} / \mathrm{s}$ (25\%: 450,479, 75\%: 500,611); however, no significant difference was found 4 weeks later: median $458,000 \mu \mathrm{m} / \mathrm{s}$ (25\%: 439,000, 75\%: 496,250). Similarly the PR dropped $8.3 \%(p<0.05)$ at the edges of the bowel strip after the spiral incision from 1.000 (25\%: $0.982,75 \%: 1.000)$ to 0.917 (25\%: $0.873,75 \%: 0.993)$, but recovered after 4 weeks to 0.980 (25\%: 0.950, 75\%: 1.000) (Fig. 2).

The Malecoth catheters were lost within 2 weeks. At the level of the epidermis the opening of the channels was found to be narrow, but after some dilatation they became patent and easily catheterisable. All implanted channels remained viable and straight. The histology showed no necrosis of mucosa and muscle layers either at the skin or bladder level.

\section{Discussion}

The concept of SILT, that is refashioning normal calibre bowel tube to a narrower shape in order to create an alternative Mitrofanoff channel, was tested in this study.

The channel length achieved by SILT in this experiment $(100 \pm 26.4 \mathrm{~mm})$ is outstanding; it exceeds the calculated length of the Monti technique (bowel width $\times 2$ ) and the double Monti and the Casele procedure (bowel width $\times 4$ ) (Fig. 3).

Theoretically, catheterisable channels could be designed by determining the length of the channel required and calculating back the length of segment needed to harvest based on the percentage increase in length and tailoring. In our model we experienced 55\% lengthening and $80 \%$ tailoring; this is in accordance with the linear regression between the lengthening and tailoring in SILT we reported earlier [5]. However, in this model it became clear that two full $\left(360^{\circ}\right)$ spiral cuts, one proximal and one distal to the mesenteric attachment selected, provide a longer channel compared with the present techniques without bulky mesentery at the end of the channels for easy implantation. The length of the harvested bowel segment is rather determined by the width of the mesentery selected. This measures $15-20 \mathrm{~mm}$ at the Monti or Casale procedures and it is usually enough to incorporate a strong branch of the mesenteric vessel. The mesentery in our model was designed to be $15 \mathrm{~mm}$ wide; however, a wider mesentery strip could have been easily used. This would have resulted in stronger mesentery and would have further increased the channel length, however beyond a certain extent may have complicated spiral reconstruction. It is more difficult to reconstruct a wide bowel strip to a narrow $(12 \mathrm{~F})$ spiral channel.

The minimum length of the harvested segment should be three times the width of mesentery we select. In our model this was $45 \mathrm{~mm}$. In the study however $60-80-\mathrm{mm}$-long segments were harvested to facilitate tissue handling and half of them were discarded. We believe that losing $40-50 \mathrm{~mm}$ of the bowel length is not significant.

Extrapolating our results to a human ileum, which is approximately $55 \mathrm{~mm}$ wide ( $\pi \times$ diameter/2) [9] this creates a spiral channel longer than $220 \mathrm{~mm}$. We believe a channel longer than this is hardly ever required.

A transient alteration in the microcirculation of the bowel immediately after the procedure was experienced. 
Red blood cell velocity

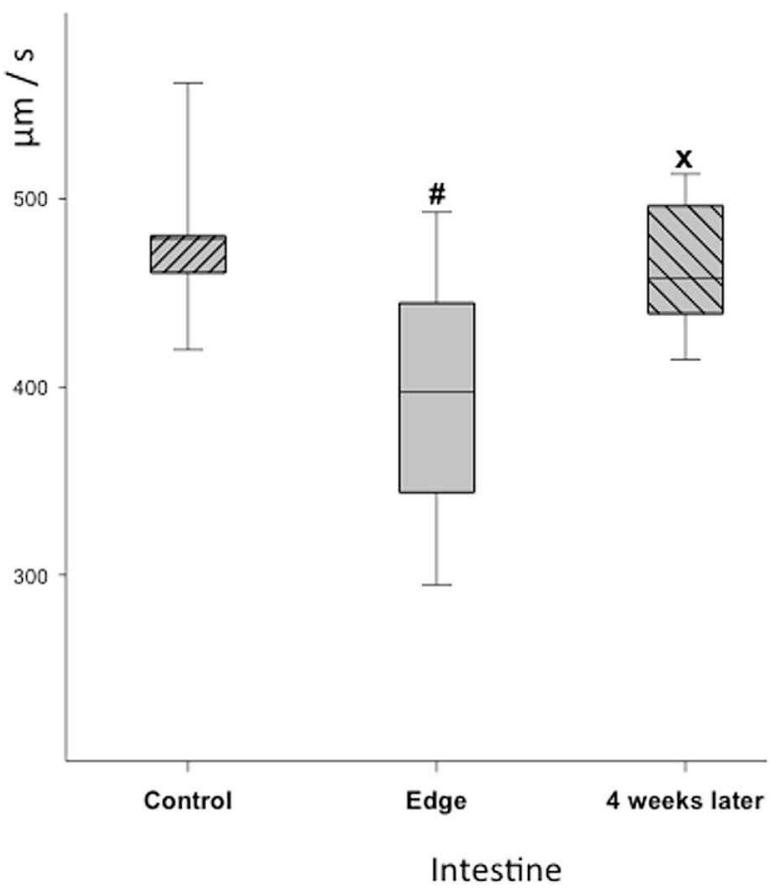

Perfusion rate

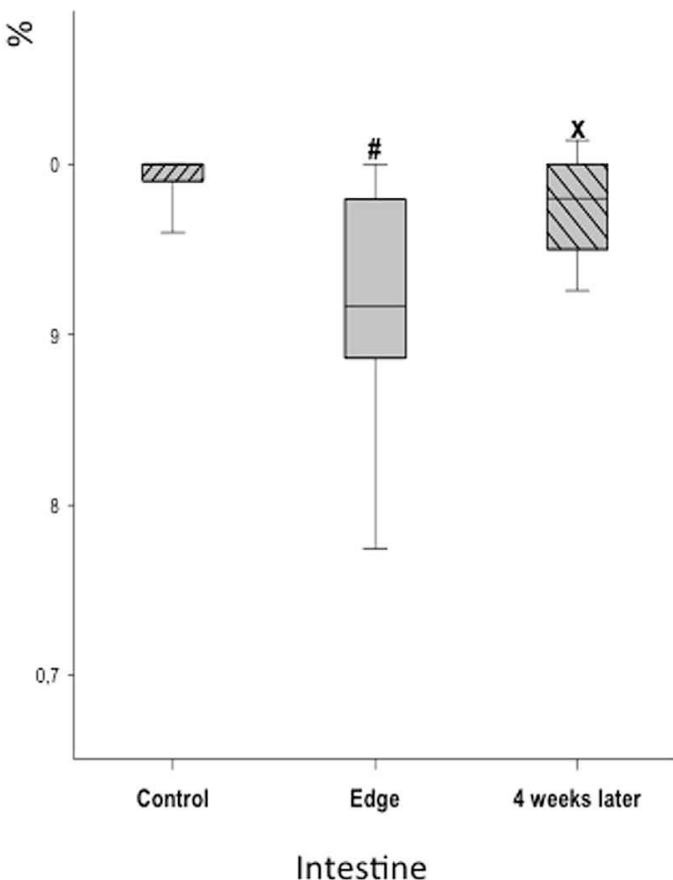

Figure 2 Data of microcirculation recorded on the disconnected bowel strip mucosa. Note. The control is the middle of the strip just below the mesentery edges. Edge is the bowel strip at primary surgery. Edge 4 weeks later is the edge of the bowel strip at the autopsy; $17 \%$ and $8.3 \%$ reduction has been measured in the median values of the red blood cell velocity and the perfusion rate at the edges of the bowel strip compared with the control at the primary surgery, but these values are recovered by the autopsy. $\# p<0.05$ vs. control; $x p<0.05$ vs. edge.

Interestingly, after using SILT in a model of short bowel syndrome, changes in the microcirculation after spiral cut were not recorded [5]. This could be explained with the fact that for the alternative Mitrofanoff the bowel was disconnected next to the vasa recta, while in the short bowel model the bowel remained continuous. Furthermore, in the short bowel model dilated $(40-50 \mathrm{~mm}$ wide) bowel

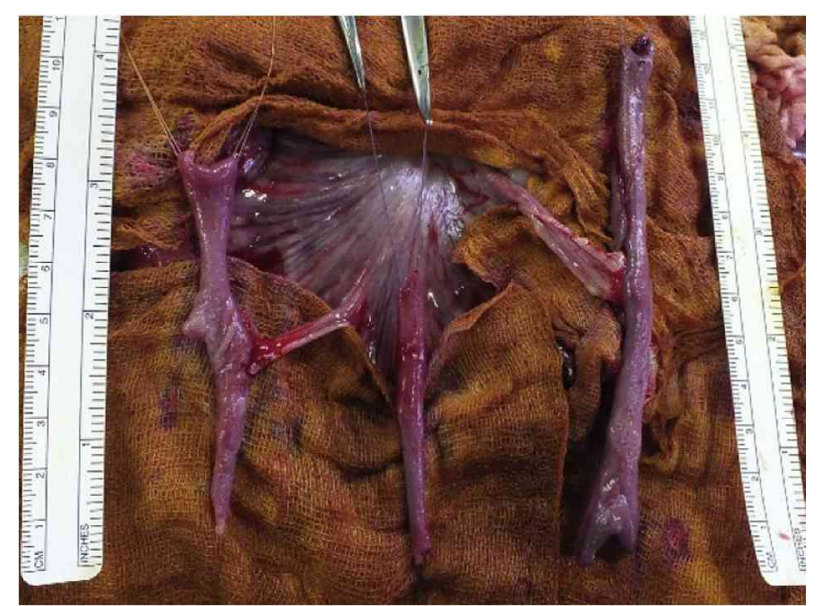

Figure 3 (Left to right) The length of the bowel strips made by the Casale, Monti, and the spiral intestinal lengthening technique. Note the angulation in the middle of the Casale strip. loop was refashioned and the bowel strip after the spiral incision was much wider $(30-40 \mathrm{~mm})$ than the present experiment where the spiral incision resulted in a $15-\mathrm{mm}$ wide bowel strip. The tissue handling could have simply had an effect on the microcirculation; however, this was performed with care and the bowel was rinsed regularly with warm saline. Four weeks later normal microcirculatory patterns were found. The channels were patent and histology did not show necrosis or atrophy.

It was possible to reconstruct the bowel strip along the long edge, as in the Monti tube. In this case the strip was kept less than $1 \mathrm{~cm}$ wide to get an ideally slim channel; however, spiral reconstruction allows cutting a wider $(1.5-2 \mathrm{~cm})$ strip with better blood supply.

In humans, catheters are usually left for a few weeks in Mitrofanoff channels and the patients self-catheterise (dilate) them on regular basis afterwards to prevent stomal stricture at the skin level. Animals do not tolerate catheters sticking out of their body (we lost our catheters within days), and intermittent catheterisation of the channels is not possible without general anaesthesia. In this study we did not expose the animals to this repeated stress. This is clearly a limitation of our model. This and the fact that pig skin is more robust than human skin might have contributed to the stomal stenosis despite the use of $\mathrm{V}$ shaped stomas in this study. However, we believe viability is the key to the reproducibility of easy catheterisation. The microcirculation data and the histology findings did not suggest chronic ischaemia at skin level. 
The Casale channels were reported to require subfascial revision in $15.2 \%$ versus $8.3 \%$ of the Monti channels [10]. The nearly double complication rate may be related to the significant angulation in the middle of the Casale channel, which has to be straightened surgically during its creation. The possible tissue memory at this point, just like the anastomosis in double Monti channels, may result in difficult catheter passage, stricture, or perforation. The spiral channels were found to be straight. The suture line within the tube runs in spiral fashion, in theory it must guide the catheter tip easily, as the barrel of a rifle guides a bullet.

In conclusion, the SILT technique is suitable on normal calibre bowel to create an alternative Mitrofanoff catheterisable stoma which is longer and straighter than can be achieved with present techniques.

This technique may have some potential for ureteral replacement as well.

\section{Ethical approval}

The experiment was approved by the Committee of Animal Research at the University Szeged (Permission no: V./1637/ 2013.).

\section{Conflict of interest}

None.

\section{Funding}

The study supported by Charitable Fund Manchester, the sponsor had no role in the study design, in the collection, analysis and interpretation of data; in the writing of the manuscript; and in the decision to submit the manuscript for publication.

\section{References}

[1] Mitrofanoff P. Trans-appendicular continent cystostomy in the management of the neurogenic bladder. Chir Pediatr 1980;21: 297.

[2] Monti PR, Lara RC, Dutra MA, de Carvalho JR. New techniques for construction of efferent conduits based on the Mitrofanoff principle. Urology 1997;49:112.

[3] Casale AJ. A long continent ileovesicostomy using a single piece of bowel. J Urol 1999;162:1743.

[4] Narayanaswamy B, Wilcox DT, Cuckow PM, Duffy PG, Ransley PG. The Yang Monti ileovesicostomy: a problematic channel? BJU Int 2001;87:861.

[5] Cserni T, Takayasu H, Muzsnay Z, Varga G, Murphy F, Folaranmi SE, et al. New idea of intestinal lengthening and tailoring. Pediatr Surg Int 2011;27:1009-13.

[6] Cserni T, Varga G, Erces D, Kaszaky J, Boros M, Morabito A, et al. Spiral intestinal lengthening and tailoring - first in vivo study. J Pediatr Surg 2013;48:1907-13.

[7] Cserni T, Biszku B, Guthy I, Dicso F, Szaloki L, Folaranmi S, et al. The first clinical application of the spiral intestinal lengthening and tailoring (SILT) in extreme short bowel syndrome. J Gastrointest Surg 2014 Jun 24;18:1852-7.

[8] Cerný V, Turek Z, Parízková R. Orthogonal polarization spectral imaging. Physiol Res 2007;56:141-7.

[9] Bannister LH. Ileum in alimentary system, Gray's anatomy. 38th ed. Churchill Livingstone; 1995.

[10] Leslie JA, Cain MP, Kaefer M, Meldrum KK, Dussinger AM, Rink RC, et al. A comparison of the Monti and Casale (spiral Monti) procedures. J Urol 2007;178:1623-7. 